\title{
Very long-term sequelae of craniopharyngioma
}

\author{
Mark Wijnen ${ }^{1,2}$, Marry M van den Heuvel-Eibrink ${ }^{2,3}$, Joseph A M J L Janssen', \\ Coriene E Catsman-Berrevoets ${ }^{4}$, Erna M C Michiels ${ }^{2}$, Marie-Lise C \\ van Veelen-Vincent ${ }^{5}$, Alof H G Dallenga ${ }^{6}$, J Herbert van den Berge ${ }^{6}$, \\ Carolien M van Rij', Aart-Jan van der Lely' and Sebastian J C M M Neggers ${ }^{\mathbf{1}, 2}$
}

${ }^{1}$ Section Endocrinology, Department of Medicine, Pituitary Centre Rotterdam, Erasmus University Medical Centre, Rotterdam, the Netherlands, '2Department of Paediatric Oncology/Haematology, Erasmus MC - Sophia Children's Hospital, Rotterdam, the Netherlands, ${ }^{3}$ Princess Maxima Centre for Paediatric Oncology, Utrecht, the Netherlands, ${ }^{4}$ Department of Paediatric Neurology, ${ }^{5}$ Department of Paediatric Neurosurgery, Erasmus MC - Sophia Children's Hospital, Rotterdam, the Netherlands, ${ }^{6}$ Department of Neurosurgery, and ${ }^{7}$ Department of Radiation Oncology, Pituitary Centre Rotterdam, Erasmus University Medical Centre, Rotterdam, the Netherlands

Correspondence should be addressed to M Wijnen Email m.wijnen.1@erasmusmc.nl

\begin{abstract}
Objective: Studies investigating long-term health conditions in patients with craniopharyngioma are limited by short follow-up durations and generally do not compare long-term health effects according to initial craniopharyngioma treatment approach. In addition, studies comparing long-term health conditions between patients with childhoodand adult-onset craniopharyngioma report conflicting results. The objective of this study was to analyse a full spectrum of long-term health effects in patients with craniopharyngioma according to initial treatment approach and age group at craniopharyngioma presentation.

Design: Cross-sectional study based on retrospective data.

Methods: We studied a single-centre cohort of 128 patients with craniopharyngioma treated from 1980 onwards (63 patients with childhood-onset disease). Median follow-up since craniopharyngioma presentation was 13 years (interquartile range: 5-23 years). Initial craniopharyngioma treatment approaches included gross total resection $(n=25)$, subtotal resection without radiotherapy $(n=44)$, subtotal resection with radiotherapy $(n=25)$, cyst aspiration without radiotherapy $(n=8)$, and ${ }^{90}$ Yttrium brachytherapy $(n=21)$.

Results: Pituitary hormone deficiencies (98\%), visual disturbances (75\%) and obesity (56\%) were the most common long-term health conditions observed. Different initial craniopharyngioma treatment approaches resulted in similar long-term health effects. Patients with childhood-onset craniopharyngioma experienced significantly more growth hormone deficiency, diabetes insipidus, panhypopituitarism, morbid obesity, epilepsy and psychiatric conditions compared with patients with adult-onset disease. Recurrence-/progression-free survival was significantly lower after initial craniopharyngioma treatment with cyst aspiration compared with other therapeutic approaches. Survival was similar between patients with childhood- and adult-onset craniopharyngioma.

Conclusions: Long-term health conditions were comparable after different initial craniopharyngioma treatment approaches and were generally more frequent in patients with childhood-compared with adult-onset disease.

() 2017 European Society of Endocrinology Printed in Great Britain
Published by Bioscientifica Ltd. 


\section{Introduction}

Craniopharyngiomas are benign (supra)sellar tumours of epithelial histology that often exhibit calcifications and fluid-filled cysts (1). They affect both children and adults, and have peak incidences between 5-9 and 40-44 years of age (2). Since their discovery in 1857 by Von Zenker (3), there has been a controversy about their aetiology, pathogenesis and optimal therapeutic approach (4). In general, craniopharyngioma treatment aims to provide long-term survival and disease control while preserving quality of life by minimizing tumourand treatment-related morbidity. This is challenging due to close proximity of the tumour to vital brain structures, like the hypothalamic-pituitary system and visual pathways (1). Craniopharyngiomas are generally treated with neurosurgical excision with or without postoperative radiotherapy. Alternative treatment options include intracystic appliance of betaemitting isotopes or chemotherapeutic substances, as well as stereotactic radiosurgery (5). Although craniopharyngioma treatment is predominantly individualized based on tumour and patient characteristics, the aggressiveness of the therapeutic approach varies from centre to centre (6). Some centres favour initial extensive excision whereby preserving hypothalamic and optic functions $(7,8,9,10,11$, $12,13,14,15)$, while other centres prefer a more conservative approach comprising limited resection or cyst decompression $(16,17,18,19,20)$.

Although recent studies on craniopharyngioma report encouraging 10-year overall survival rates between 40 and $95 \%(10,21,22,23,24,25)$, serious long-term health conditions related to tumour and treatment are frequent $(10,20,22,23,24,26,27,28,29,30,31)$, and significantly impair quality of life $(28,32)$. Most studies assessing longterm health effects in patients with craniopharyngioma are limited by short follow-up durations of less than ten years $(10,20,22,23,24,25,26,27,32,33,34)$. Furthermore, only a few studies compare long-term health conditions according to initial craniopharyngioma treatment approach $(17,35,36,37)$. Moreover, studies comparing long-term health effects between patients with childhood- and adult-onset craniopharyngioma report conflicting results $(28,30,36)$.

The objective of the present study is to analyse a full spectrum of long-term health conditions in patients with craniopharyngioma according to initial treatment approach and age group at craniopharyngioma presentation.

\section{Subjects and methods}

\section{Participants}

Patients who were diagnosed with and/or treated for craniopharyngioma at the Erasmus University Medical Centre were eligible for participation in this crosssectional study based on retrospective data. In order to reduce heterogeneity in our study population attributable to time-related differences in craniopharyngioma treatment, neuroimaging and supportive care, we chose to include only patients with craniopharyngioma treated from 1980 onwards. Our local institutional review board approved this study and all patients gave their informed consent.

\section{Data collection}

Patients were identified by a computer-based search in the electronic patient files. This search yielded 145 patients with craniopharyngioma of whom 128 were treated from 1980 onwards. Of these 128 patients, 63 were $<18$ years of age (i.e. childhood-onset), and 65 were $\geq 18$ years of age (i.e. adult-onset). Patients presenting with craniopharyngioma between 1978 and 2015 were followed-up for a median of 13 years (interquartile range: 5-23 years). Diagnoses of all but three craniopharyngiomas were histologically verified. Data regarding baseline characteristics, presenting symptoms, hydrocephalus, tumour localization and consistency, treatment, long-term health conditions and survival were extracted from the medical records.

\section{Craniopharyngioma localization and characteristics}

Data regarding craniopharyngioma localization and consistency at diagnosis were obtained from neuroimaging and neurosurgery reports. Craniopharyngioma localization was defined as intrasellar, suprasellar or both intra- and suprasellar. Craniopharyngioma consistency was characterized as unicystic, multicystic or solid. Computed tomography and magnetic resonance imaging became available in our institution in 1976 and 1990 respectively.

\section{Craniopharyngioma treatment}

Initial craniopharyngioma treatment approaches were subdivided in gross total resection $(n=25)$, subtotal 
resection without radiotherapy $(n=44)$, subtotal resection with radiotherapy $(n=25)$, cyst aspiration without radiotherapy $(n=8)$ and ${ }^{90}$ Yttrium brachytherapy $(n=21)$. Three patients were treated with neurosurgical excision but had an unknown degree of resection and two patients were treated with other therapeutic approaches (radiotherapy only $(n=1)$, cyst aspiration with radiotherapy $(n=1))$. These latter five patients were excluded from the analyses according to initial craniopharyngioma treatment approach. Gross total resection was defined as the complete removal of the craniopharyngioma as documented by the neurosurgeon, without residual disease on postoperative imaging. In each patient, the initial therapeutic approach was based on the treating physicians' preferences. Since the nineties, all patients were systematically discussed in multidisciplinary meetings by specialists in (paediatric) neurosurgery, (paediatric) endocrinology, paediatric neurology, paediatric oncology, radiation-oncology and neuroradiology.

\section{Long-term health conditions}

Visual disturbances, epilepsy requiring treatment, neurological deficits, cognitive impairment, behavioural changes, psychiatric conditions requiring treatment (postoperative delirium excluded), weight status, obstructive sleep apnoea, pituitary hormone deficiencies, secondary neoplasms and cardio- and cerebrovascular events were studied as outcomes reflecting long-term health conditions. Visual disturbances were diagnosed based on ophthalmic examinations. Visual acuity was determined after correction for refraction disorders. Visual field defects were diagnosed with Goldmann perimetry. Neurological deficits were classified into two categories (i.e. mild and severe) based on their reported impact on independent daily living. Deficits that had no impact were classified as mild and deficits that compromised independent daily living were classified as severe. Cognitive impairment and behavioural changes were considered to be present if documented in the patient files. Weight status was classified according to body mass index. Obstructive sleep apnoea was diagnosed by polysomnography in patients with suspected sleep disordered breathing. Pituitary hormone deficiencies were confirmed by formal pituitary function testing in all patients. In ten patients, the pituitary gland was completely removed during neurosurgical excision of the craniopharyngioma.

\section{Surviva}

Overall and recurrence-/progression-free survival were calculated since initial craniopharyngioma treatment. Craniopharyngioma recurrence/progression was diagnosed with neuroimaging. Recurrence was defined as reappearance of the craniopharyngioma after gross total resection. Progression was defined as re-growth of the craniopharyngioma after subtotal resection, irradiation, cyst aspiration or ${ }^{90}$ Yttrium brachytherapy. Follow-up neuroimaging was performed regularly, based on physician's preference and clinical judgement. The cause of death was classified as directly related to the craniopharyngioma itself or not.

\section{Statistical analysis}

Statistical analyses were performed using the Statistical Package for Social Sciences (SPSS 24). Continuous variables were described as median and interquartile range; discrete variables as frequencies and percentages. Evaluations were based on the number of patients with available data. Comparisons according to initial craniopharyngioma treatment approach were performed by one-way analyses of variance for continuous variables and Chi-squared tests for discrete variables. In case the assumptions were not met, Kruskal-Wallis tests and likelihood ratio statistics were used. Comparisons between patients with childhood- and adult-onset craniopharyngioma were made by Student's t-test for continuous variables and Chi-squared tests for discrete variables. Mann-Whitney $U$ tests and likelihood ratio statistics or Fisher's exact tests were used in case the assumptions were not met. Overall and recurrence-/ progression-free survival were estimated by Kaplan-Meier methods. Log-rank tests were used to compare survival curves. In case statistical significance was reached in a variable concerning more than two groups, Bonferroni-corrected post hoc analyses were performed to specify subgroup-related differences. Because certain long-term health conditions may only become apparent after a prolonged follow-up period, and because craniopharyngioma management may have evolved over time due to advances in treatment, neuroimaging and supportive care, we investigated the influence of the treatment decade (i.e. 1980-1989, 19901999, 2000-2009, or $\geq 2010$ ) and follow-up duration since initial craniopharyngioma treatment on the development of long-term health effects using multivariable logistic regression models including treatment decade and follow-up duration as independent variables. We investigated the influence of treatment decade on survival by log-rank 
tests for trend. A $P$-value $<0.05$ (two-tailed) was considered statistically significant.

\section{Results}

\section{Presenting symptoms}

Presenting symptoms in patients with craniopharyngioma are shown in Table 1. Visual disturbances, pituitary hormone deficiencies and headaches were the most common disease manifestations and occurred in 87, 71, and $68 \%$ of the patients respectively. Hypogonadotropic hypogonadism was the most common pituitary hormone deficiency and it affected $60 \%$ of the patients. Diabetes insipidus was found in $7 \%$ of the patients. Underweight and obesity affected $10 \%$ and $12 \%$ of the patients respectively. Two patients (both with childhood-onset craniopharyngioma) presented with blindness. Patients with childhood-onset craniopharyngioma presented significantly more growth hormone deficiency (53\% vs $27 \%, P<0.01)$ compared with patients with adult-onset craniopharyngioma. Visual field defects (85\% vs 59\%, $P<0.01)$, cognitive impairment $(16 \%$ vs $3 \%, P=0.02)$, overweight $(39 \%$ vs $16 \%, P<0.01)$ and secondary hypothyroidism (56\% vs 32\%, $P<0.01)$ were significantly more common in patients with adult- compared with childhood-onset craniopharyngioma.

\section{Craniopharyngioma characteristics at diagnosis}

Tumour characteristics at diagnosis are depicted in Table 1. Tumour consistency was significantly different between patients with childhood- and adult-onset craniopharyngioma. Bonferroni-corrected post hoc analyses revealed a significantly higher number of multicystic craniopharyngiomas in patients with childhood- compared with adult-onset disease (45\% vs $21 \% ; P=0.02$ ). Hydrocephalus, as well as hypothalamic and/or third ventricle involvement were also significantly more common in patients with childhoodcompared with adult-onset craniopharyngioma (40\% vs $18 \%, P<0.01 ; 66 \%$ vs $42 \%, P=0.01)$. Patient and tumour characteristics were not significantly different between different initial craniopharyngioma treatment approaches (Table 2).

\section{Craniopharyngioma treatment}

In the patients who were initially treated with neurosurgical excision, the neurosurgical approach was transsphenoidal in 35 patients (39\%), subfrontal in 31 patients (35\%), pterional in 20 patients $(23 \%)$, transcallosal in two patients (2\%) and supra-orbital in one patient (1\%). In five patients $(5 \%)$ data regarding the neurosurgical approach were missing. There were no significant differences in the neurosurgical approaches used between patients treated with gross total resection, subtotal resection without radiotherapy and subtotal resection with radiotherapy. Initial craniopharyngioma treatment approaches, as well as neurosurgical approaches were not significantly different between patients with childhood- and adult-onset craniopharyngioma. In the patients who initially received radiotherapy, conventional external beam radiotherapy was applied to eight patients and stereotactic radiotherapy to 16 patients. In four patients, detailed information on the type of radiotherapy was missing. In the patients who were initially treated with radiotherapy, the median cumulative radiation dose was $54 \mathrm{~Gy}$ (interquartile range: 54-54 Gy); delivered in a median 30 fractions (interquartile range: 30-30 fractions). The median duration between neurosurgery and radiotherapy was two months (interquartile range: 1-6 months).

In our institution, initial craniopharyngioma treatment changed over time (Fig. 1). The use of gross total resection declined from 22\% during 1980-1989 to $12 \%$ in $\geq 2010$. Meanwhile, the use of subtotal resection (with or without radiotherapy) increased from 30\% during 1980-1989 to $71 \%$ in $\geq 2010$. ${ }^{90}$ Yttrium brachytherapy was predominantly used during 1980-1989.

\section{Long-term health conditions}

Long-term health conditions in patients with craniopharyngioma are shown in Table 3. Pituitary hormone deficiencies, visual disturbances and obesity were the most common long-term health effects and affected 98, 75 and 56\% of the patients respectively. Hypogonadotropic hypogonadism was the most common pituitary hormone deficiency and was found in $92 \%$ of the patients. Diabetes insipidus was diagnosed in $61 \%$ of the patients. Five patients demonstrated blindness (four after initial treatment with subtotal resection; one after initial treatment with ${ }^{90}$ Yttrium brachytherapy). Severe neurological deficits (i.e. compromising independent daily living) affected 11 patients. In three patients, severe neurological deficits were directly related to the craniopharyngioma itself. One patient developed radiation encephalopathy six years after conventional external beam radiotherapy at an age of 41 years. Three 
Table 1 Baseline characteristics, presenting symptoms, and tumour characteristics at diagnosis. Data are presented as $n$ (\%).

\begin{tabular}{|c|}
\hline Baseline characteristics \\
\hline$n$ \\
\hline Male \\
\hline Female \\
\hline Age (year) at presentation, median (IQR) \\
\hline Presenting symptoms \\
\hline Headaches \\
\hline Visual acuity disorders \\
\hline Visual field defects \\
\hline Epilepsy \\
\hline Neurological deficits \\
\hline Altered level of consciousness \\
\hline Cognitive impairment \\
\hline Behavioural changes \\
\hline History of weight change \\
\hline Increase \\
\hline Decrease \\
\hline Weight status (BMI $\left.\left(\mathrm{kg} / \mathrm{m}^{2}\right)\right)$ \\
\hline Underweight $(<18.5)$ \\
\hline Normal weight (18.5-24.9) \\
\hline Overweight (25.0-29.9) \\
\hline Class I obesity (30.0-34.9) \\
\hline Class II obesity (35.0-39.9) \\
\hline Class III obesity $(\geq 40.0)$ \\
\hline Class I-III obesity $(\geq 30.0)$ \\
\hline Pituitary hormone deficiencies \\
\hline $\mathrm{GH}$ \\
\hline $\mathrm{FSH} / \mathrm{LH}$ \\
\hline АСTH \\
\hline TSH \\
\hline $\mathrm{ADH}$ \\
\hline Anterior $\mathrm{PH}$ \\
\hline Complete $\mathrm{PH}$ \\
\hline Tumour characteristics \\
\hline Localization \\
\hline Intrasellar \\
\hline Suprasellar \\
\hline Intra-/suprasellar \\
\hline Consistency \\
\hline Unicystic \\
\hline Multicystic \\
\hline Solid \\
\hline Presence of hydrocephalus \\
\hline Hypothalamic and/or third ventricle involvement \\
\hline Brain stem compression \\
\hline
\end{tabular}

\begin{tabular}{|c|c|}
\hline All craniopharyngiomas & Childhood-onset \\
\hline 128 & 63 \\
\hline $57(45)$ & $25(40)$ \\
\hline $71(56)$ & $38(60)$ \\
\hline $19(8-42)$ & $8(5-12)$ \\
\hline $83(68)$ & $42(71)$ \\
\hline $73(73)$ & $33(72)$ \\
\hline $72(74)$ & $23(59)$ \\
\hline $7(6)$ & $6(10)$ \\
\hline $42(34)$ & $25(42)$ \\
\hline $26(21)$ & $15(25)$ \\
\hline $12(10)$ & $2(3)$ \\
\hline $15(12)$ & $8(14)$ \\
\hline $13(11)$ & $3(5)$ \\
\hline $19(16)$ & $9(15)$ \\
\hline $10(10)$ & $8(16)$ \\
\hline $51(51)$ & $31(61)$ \\
\hline $27(27)$ & $8(16)$ \\
\hline $7(7)$ & $2(4)$ \\
\hline $2(2)$ & $0(0)$ \\
\hline $3(3)$ & $2(4)$ \\
\hline $12(12)$ & $4(8)$ \\
\hline $87(71)$ & $40(68)$ \\
\hline $48(40)$ & $31(53)$ \\
\hline $49(60)$ & $10(56)$ \\
\hline $42(34)$ & $17(28)$ \\
\hline $54(44)$ & $19(32)$ \\
\hline $8(7)$ & $4(7)$ \\
\hline $17(14)$ & $10(17)$ \\
\hline $4(3)$ & $2(3)$ \\
\hline $5(4)$ & $3(5)$ \\
\hline $48(39)$ & $23(38)$ \\
\hline $70(57)$ & $34(57)$ \\
\hline $71(61)$ & $29(52)$ \\
\hline $38(33)$ & $25(45)$ \\
\hline $8(7)$ & $2(4)$ \\
\hline $36(29)$ & $25(40)$ \\
\hline $60(53)$ & $35(66)$ \\
\hline $16(14)$ & $6(11)$ \\
\hline
\end{tabular}

\begin{tabular}{|c|c|}
\hline Adult-onset & P-value* \\
\hline \multicolumn{2}{|l|}{65} \\
\hline 32 (49) & 0.28 \\
\hline \multicolumn{2}{|l|}{$33(51)$} \\
\hline $41(29-55)$ & $<0.01$ \\
\hline $41(65)$ & 0.47 \\
\hline $40(74)$ & 0.79 \\
\hline $49(85)$ & $<0.01$ \\
\hline $1(2)$ & 0.06 \\
\hline $17(27)$ & 0.07 \\
\hline $11(18)$ & 0.28 \\
\hline $10(16)$ & 0.02 \\
\hline \multirow[t]{2}{*}{$7(11)$} & 0.68 \\
\hline & 0.14 \\
\hline \multicolumn{2}{|l|}{$10(16)$} \\
\hline \multicolumn{2}{|l|}{$10(16)$} \\
\hline $2(4)$ & 0.09 \\
\hline $20(41)$ & 0.05 \\
\hline $19(39)$ & $<0.01$ \\
\hline $5(10)$ & 0.26 \\
\hline $2(4)$ & 0.24 \\
\hline $1(2)$ & 1.00 \\
\hline $8(16)$ & 0.19 \\
\hline $47(73)$ & 0.49 \\
\hline $17(27)$ & $<0.01$ \\
\hline $39(61)$ & 0.68 \\
\hline 25 (39) & 0.21 \\
\hline $35(56)$ & $<0.01$ \\
\hline $4(6)$ & 1.00 \\
\hline 7 (12) & 0.39 \\
\hline \multirow[t]{2}{*}{$2(3)$} & 1.00 \\
\hline & 0.87 \\
\hline \multicolumn{2}{|l|}{$2(3)$} \\
\hline \multicolumn{2}{|l|}{$25(40)$} \\
\hline \multicolumn{2}{|l|}{$36(57)$} \\
\hline & 0.02 \\
\hline \multicolumn{2}{|l|}{$42(69)$} \\
\hline \multicolumn{2}{|l|}{$13(21)$} \\
\hline \multicolumn{2}{|l|}{$6(10)$} \\
\hline $11(18)$ & $<0.01$ \\
\hline $25(42)$ & 0.01 \\
\hline $10(17)$ & 0.39 \\
\hline
\end{tabular}

Data regarding headaches, epilepsy, neurological deficits, altered level of consciousness, cognitive impairment, behavioural changes and weight change were missing in 6 patients. Data regarding visual acuity disorders and visual field defects were missing in 28 and 31 patients respectively. Weight status could not be determined in 28 patients. Data regarding pituitary hormone deficiencies were missing in 8 patients (growth hormone (GH) in 7 patients, follicle stimulating hormone/luteinizing hormone ( $\mathrm{FSH} / \mathrm{LH})$ in 4 patients, adrenocorticotropic hormone (ACTH) in 4 patients, thyroid stimulating hormone (TSH) in 5 patients, antidiuretic hormone (ADH) in 4 patients, and anterior and complete panhypopituitarism (PH) in 8 patients). Also, 42 patients were too young to reliably assess gonadal function (girls $<14$ years of age; boys $<15$ years of age). Data regarding initial craniopharyngioma localization, consistency, hydrocephalus, hypothalamic and/or third ventricle involvement, and brain stem compression were missing in 5, 11, 3, 15 and 14 patients respectively.

$\mathrm{BMI}$, body mass index; IQR, interquartile range.

*Childhood- vs adult-onset

patients suffered from radionecrosis related to leakage of the installed radio-isotope a median 15 months after ${ }^{90}$ Yttrium brachytherapy at a median age of 54 years. In four patients, cerebrovascular events caused severe neurological dysfunction a median seven months after initial craniopharyngioma treatment at a median age of 27 years. This latter group included one child who experienced a cerebral infarction of the entire left 
Table 2 Patient characteristics and tumour characteristics at diagnosis according to initial craniopharyngioma treatment. Data are presented as $n(\%)$.

\begin{tabular}{l} 
Baseline characteristics \\
\hline$n$ \\
Male \\
Female \\
Age (year) at presentation, median (IQR) \\
Tumour characteristics \\
$\quad$ Localization \\
$\quad$ Intrasellar \\
$\quad$ Suprasellar \\
$\quad$ Intra-/suprasellar \\
Consistency \\
$\quad$ Unicystic \\
$\quad$ Multicystic \\
$\quad$ Solid \\
Presence of hydrocephalus \\
Hypothalamic and/or third ventricle involvement \\
Brain stem compression
\end{tabular}

\begin{tabular}{c}
\hline $\mathbf{G T R}$ \\
\hline 25 \\
$9(36)$ \\
$16(64)$ \\
$15(8-37)$
\end{tabular}

\begin{tabular}{c}
\hline STR w/o RTx \\
\hline 44 \\
$19(43)$ \\
$25(57)$ \\
$26(9-41)$
\end{tabular}

\begin{tabular}{l} 
STR with RTx \\
\hline 25 \\
$11(44)$ \\
$14(56)$ \\
$26(15-46)$
\end{tabular}

\begin{tabular}{c}
\hline Cyst aspiration \\
\hline 8 \\
$3(38)$ \\
$5(63)$ \\
$13(5-71)$
\end{tabular}

\begin{tabular}{|c|c|}
\hline${ }^{90}$ Yttrium & va \\
\hline 21 & \\
\hline $\begin{array}{r}12(57) \\
9(43)\end{array}$ & 0.69 \\
\hline $11(6-45)$ & 0.3 \\
\hline
\end{tabular}

$\begin{array}{cc}1(4) & 1(2) \\ 9(39) & 14(33) \\ 13(57) & 28(65) \\ & \\ 15(65) & 23(58) \\ 7(30) & 14(35) \\ 1(4) & 3(8) \\ 6(25) & 10(23) \\ 11(48) & 20(50) \\ 2(9) & 4(10)\end{array}$

$1(4)$
$9(38)$
$14(58)$

$15(65)$
$6(26)$
$2(9)$
$5(21)$
$15(68)$
$4(17)$

$0(0)$

$5(63)$

3 (38)

10 (48)

10 (48)

$5(63)$

3 (38)

13 (65)

7 (35)

0 (0)

7 (33)

5 (63)

6 (75)

$1(13)$

Data regarding initial craniopharyngioma localization, consistency, hydrocephalus, hypothalamic and/or third ventricle involvement, and brain stem compression were missing in 4, 9, 3, 13 and 12 patients respectively.

IQR, interquartile range; ${ }^{90}$ Yttrium, ${ }^{90}$ Yttrium brachytherapy; GTR, gross total resection; RTx, radiotherapy; STR, subtotal resection; w/o, without.

hemisphere without a known cause approximately two years after craniopharyngioma resection.

Long-term health conditions according to initial craniopharyngioma treatment are shown in Table 4. Initial craniopharyngioma treatment with gross total resection, subtotal resection with or without radiotherapy, cyst aspiration and ${ }^{90} \mathrm{Yttrium}$ brachytherapy resulted in a similar pattern of long-term health effects. Long-term health conditions in patients with childhood- compared with adult-onset craniopharyngioma are shown in Table 3. Patients with childhood-onset craniopharyngioma demonstrated significantly more growth hormone deficiency ( $93 \%$ vs 68\%, $P<0.01$ ), diabetes insipidus $(79 \%$

\section{Craniopharyngioma therapy per treatment decade}

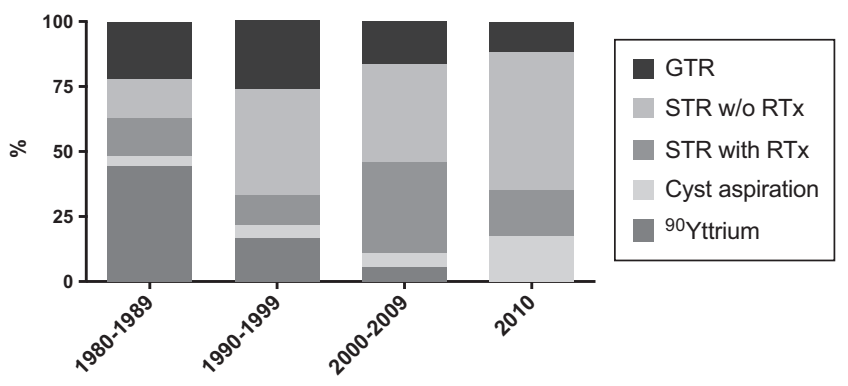

\section{Figure 1}

Initial craniopharyngioma treatment per decade. \%, percentage; ${ }^{90}$ Yttrium, ${ }^{90}$ Yttrium brachytherapy; GTR, gross total resection; RTx, radiotherapy; STR, subtotal resection; w/o, without. vs $43 \%, P<0.01)$, anterior panhypopituitarism $(79 \%$ vs $61 \%, P=0.03$ ), complete panhypopituitarism (66\% vs $34 \%$, $P<0.01$ ), morbid obesity ( $24 \%$ vs $6 \%, P<0.01$ ), epilepsy (32\% vs $15 \%, P=0.03$ ) and psychiatric conditions (56\% vs $23 \%, P<0.01)$ compared with patients with adult-onset craniopharyngioma. Cardio- and cerebrovascular events were significantly more common in patients with adultcompared with childhood-onset craniopharyngioma ( $26 \%$ vs $5 \%, P<0.01$ ).

Initial craniopharyngioma treatment in more recent decades resulted in significant reductions of morbid obesity (odds ratio (OR): 0.3, 95\% confidence interval $(\mathrm{CI}): 0.12-0.86 ; P=0.02)$ and severe neurological deficits (OR: $0.4,95 \%$ CI: 0.21-0.97; $P=0.04$ ) after adjustment for follow-up duration. In addition, a prolonged follow-up duration after craniopharyngioma presentation was associated with a significantly lower risk of severe neurological deficits after adjustment for treatment decade (OR: 0.9, 95\% CI: 0.81-0.98; $P=0.02$ ).

\section{Survival}

Overall and recurrence-/progression-free survival after initial craniopharyngioma treatment are shown in Figs 2 and 3. Overall survival was 93,85 and $77 \%$ at 5, 10 and 20 years of follow-up. Recurrence-/progressionfree survival was 90,74 and $52 \%$ at 5, 10 and 20 years of follow-up. Although overall survival was comparable after initial craniopharyngioma treatment with gross total resection, subtotal resection with or without radiotherapy, 
Table 3 Long-term health conditions in patients with craniopharyngioma.

\begin{tabular}{|c|c|c|c|c|}
\hline & All craniopharyngiomas & Childhood-onset & Adult-onset & $P$-value* * \\
\hline$n$ & 128 & 63 & 65 & \\
\hline Follow-up (years) since presentation, median (IQR) & $13(5-23)$ & $14(5-23)$ & $11(6-23)$ & 0.81 \\
\hline Age (years) at follow-up, median (IQR) & $37(21-54)$ & $22(15-31)$ & $54(44-70)$ & $<0.01$ \\
\hline Visual acuity disorders & $71(63)$ & $34(63)$ & $37(64)$ & 0.93 \\
\hline Visual field defects & $65(66)$ & $31(66)$ & $34(65)$ & 0.95 \\
\hline Epilepsy & $30(23)$ & $20(32)$ & $10(15)$ & 0.03 \\
\hline Neurological deficits: & $30(23)$ & $16(25)$ & $14(22)$ & 0.61 \\
\hline Mild & $19(15)$ & $10(16)$ & $9(14)$ & 0.75 \\
\hline Severe & $11(9)$ & $6(10)$ & $5(8)$ & 0.71 \\
\hline Cognitive impairment & $36(28)$ & $22(35)$ & $14(22)$ & 0.09 \\
\hline Behavioural changes & $24(19)$ & 12 (19) & 12 (19) & 0.93 \\
\hline Psychiatric conditions & $50(39)$ & $35(56)$ & $15(23)$ & $<0.01$ \\
\hline \multicolumn{5}{|l|}{ Weight status (BMI $\left.\left(\mathrm{kg} / \mathrm{m}^{2}\right)\right)$} \\
\hline Underweight $(<18.5)$ & $4(4)$ & $4(7)$ & $0(0)$ & 0.12 \\
\hline Normal weight (18.5-24.9) & $18(16)$ & $7(12)$ & $11(21)$ & 0.22 \\
\hline Overweight (25.0-29.9) & $27(24)$ & $10(17)$ & $17(32)$ & 0.07 \\
\hline Class I obesity (30.0-34.9) & $34(31)$ & $18(31)$ & $16(30)$ & 0.92 \\
\hline Class II obesity (35.0-39.9) & $11(10)$ & $5(9)$ & $6(11)$ & 0.63 \\
\hline Class III obesity $(\geq 40.0)$ & $17(15)$ & $14(24)$ & $3(6)$ & $<0.01$ \\
\hline Class I-III obesity $(\geq 30.0)$ & $62(56)$ & $37(64)$ & 25 (47) & 0.08 \\
\hline Obstructive sleep apnoea & $11(9)$ & $7(11)$ & $4(6)$ & 0.32 \\
\hline Pituitary hormone deficiencies: & $125(98)$ & $61(97)$ & $64(99)$ & 0.62 \\
\hline $\mathrm{GH}$ & $99(81)$ & $57(93)$ & $42(68)$ & $<0.01$ \\
\hline $\mathrm{FSH} / \mathrm{LH}$ & $110(92)$ & $49(91)$ & $61(94)$ & 0.73 \\
\hline ACTH & $111(87)$ & $54(86)$ & $57(88)$ & 0.74 \\
\hline TSH & $116(91)$ & $58(92)$ & $58(91)$ & 0.77 \\
\hline $\mathrm{ADH}$ & $78(61)$ & $50(79)$ & $28(43)$ & $<0.01$ \\
\hline Anterior $\mathrm{PH}$ & $85(70)$ & $48(79)$ & $37(61)$ & 0.03 \\
\hline Complete $\mathrm{PH}$ & $63(50)$ & $41(66)$ & $22(34)$ & $<0.01$ \\
\hline Secondary neoplasms* & $22(17)$ & $7(11)$ & $15(23)$ & 0.07 \\
\hline Cardio- and/or cerebrovascular events & $20(16)$ & $3(5)$ & $17(26)$ & $<0.01$ \\
\hline
\end{tabular}

Data regarding visual acuity disorders and visual field defects were missing in 16 and 29 patients respectively. Weight status could not be determined in 17 patients. Data regarding pituitary hormone deficiencies were missing in 6 patients (GH in 5 patients, FSH/LH in 1 patient, TSH in 1 patient, anterior PH in 6 patients, and complete PH in 1 patient). Also, 8 patients were too young to reliably assess gonadal function.

*Secondary neoplasms included meningioma $(n=2)$, prostate cancer $(n=2)$, breast cancer $(n=2)$, arachnoid cyst $(n=2)$, thyroglossal duct cyst $(n=1)$, fibroadenoma $(n=2)$, cavernous sinus schwannoma $(n=1)$, growth hormone-producing pituitary adenoma $(n=1)$, pineal gland cyst $(n=1)$, pancreatic cancer $(n=1)$, lung cancer $(n=1)$, mesothelioma $(n=1)$, bladder cancer $(n=1)$, ovarian cancer $(n=1)$, cervical cancer $(n=1)$, calcifying odontogenic cyst $(n=1)$, osteoma of the skull $(n=1)$, haemangioma $(n=1)$, Hodgkin lymphoma $(n=1)$ and neuro-endocrine tumour of the appendix $(n=1)$.

**Childhood vs adult-onset

BMI, body mass index; IQR, interquartile range.

cyst aspiration and ${ }^{90}$ Yttrium brachytherapy, recurrence- $/$ progression-free survival was not $(P=0.03)$. Bonferronicorrected post hoc analyses revealed a significantly lower recurrence-/progression-free survival after initial craniopharyngioma treatment with cyst aspiration compared with the other treatment approaches $(P=0.01)$. Overall and recurrence-/progression-free survival were similar in patients with childhood- and adult-onset craniopharyngioma. Patients with craniopharyngioma recurrence/progression experienced a significantly lower overall survival compared with patients without craniopharyngioma recurrence/progression $(P<0.01)$. Initial craniopharyngioma treatment in more recent decades resulted in similar overall survival $(P=0.50$ for trend) and lower recurrence-/progression-free survival $(P<0.01$ for trend). Overall survival was also similar over time in the subgroup of patients with craniopharyngioma recurrence/progression ( $P=0.46$ for trend).

In our study, 22 patients (17\%) died during follow-up. In five patients (23\%) the cause of death was directly related to the craniopharyngioma itself (tumour invading vital brain structures $(n=4)$, brain herniation $(n=1))$. Eleven patients $(50 \%)$ died of causes not directly related to the craniopharyngioma itself (infection $(n=4)$, cardiac arrest $(n=2)$, secondary malignancies $(n=3)$, cerebrovascular infarction $(n=1)$, radiation encephalopathy $(n=1))$. The cause of death was unknown in six patients (27\%). Deaths directly related to the craniopharyngioma itself 
Table 4 Long-term health conditions according to initial craniopharyngioma treatment. Data represented as $n$ (\%).

\begin{tabular}{|c|c|c|c|c|c|c|}
\hline & GTR & STR w/o RTX & STR with RTX & Cyst aspiration & ${ }^{90} Y t t r i u m$ & $P$-value \\
\hline$n$ & 25 & 44 & 25 & 8 & 21 & 0.13 \\
\hline Follow-up (years), median (IQR) & $14(6-21)$ & $9(5-23)$ & $10(6-14)$ & $12(2-24)$ & $22(9-29)$ & 0.13 \\
\hline Age (years) at follow-up, median (IQR) & $30(18-51)$ & $42(21-60)$ & $40(21-65)$ & $37(18-74)$ & $35(28-56)$ & 0.72 \\
\hline Visual acuity disorders & $10(50)$ & $24(63)$ & $14(58)$ & $5(71)$ & $16(84)$ & 0.20 \\
\hline Visual field defects & $12(67)$ & $19(61)$ & $11(52)$ & $5(71)$ & $17(94)$ & 0.07 \\
\hline Epilepsy & $6(24)$ & $10(23)$ & $3(12)$ & $2(25)$ & $8(38)$ & 0.36 \\
\hline Neurological deficits & $6(24)$ & $8(18)$ & $5(20)$ & $1(13)$ & $9(43)$ & 0.25 \\
\hline Mild & $3(12)$ & $6(14)$ & $3(12)$ & $1(13)$ & $5(24)$ & 0.81 \\
\hline Severe & $3(12)$ & $2(5)$ & $2(8)$ & $0(0)$ & $4(19)$ & 0.28 \\
\hline Cognitive impairment & $11(44)$ & $7(16)$ & $9(36)$ & $2(25)$ & $7(33)$ & 0.11 \\
\hline Behavioural changes & $4(16)$ & $9(21)$ & $7(28)$ & $2(25)$ & $2(10)$ & 0.56 \\
\hline Psychiatric conditions & $8(32)$ & $18(41)$ & $9(36)$ & $5(63)$ & $8(38)$ & 0.65 \\
\hline \multicolumn{7}{|l|}{ Weight status (BMI $\left.\left(\mathrm{kg} / \mathrm{m}^{2}\right)\right)$} \\
\hline Underweight $(<18.5)$ & $0(0)$ & $3(8)$ & $1(4)$ & $0(0)$ & $0(0)$ & 0.28 \\
\hline Normal weight (18.5-24.9) & $5(22)$ & $6(16)$ & $4(17)$ & $1(13)$ & $2(13)$ & 0.95 \\
\hline Overweight (25.0-29.9) & $7(30)$ & $10(27)$ & $4(17)$ & $2(25)$ & $3(19)$ & 0.82 \\
\hline Class I obesity (30.0-34.9) & $5(22)$ & $11(30)$ & $8(35)$ & $2(25)$ & $6(38)$ & 0.82 \\
\hline Class II obesity (35.0-39.9) & $2(9)$ & $2(5)$ & $3(13)$ & $2(25)$ & $2(13)$ & 0.58 \\
\hline Class III obesity $(\geq 40.0)$ & $4(17)$ & $5(14)$ & $3(13)$ & $1(13)$ & $3(19)$ & 0.98 \\
\hline Class I-III obesity $(\geq 30.0)$ & $11(48)$ & $18(49)$ & $14(61)$ & $5(63)$ & $11(69)$ & 0.58 \\
\hline Obstructive sleep apnoea & $3(12)$ & $5(11)$ & $1(4)$ & $1(13)$ & $1(5)$ & 0.71 \\
\hline Pituitary hormone deficiencies: & $23(92)$ & $44(100)$ & $25(100)$ & $7(88)$ & $21(100)$ & 0.08 \\
\hline $\mathrm{GH}$ & $19(79)$ & $34(77)$ & $20(80)$ & $5(63)$ & $17(94)$ & 0.33 \\
\hline FSH/LH & $21(88)$ & $38(95)$ & $22(92)$ & $6(86)$ & $18(95)$ & 0.46 \\
\hline ACTH & $22(88)$ & $40(91)$ & $22(88)$ & $6(75)$ & $17(81)$ & 0.71 \\
\hline TSH & $22(88)$ & 41 (95) & $22(88)$ & $6(75)$ & $20(95)$ & 0.41 \\
\hline $\mathrm{ADH}$ & $17(68)$ & $30(68)$ & $13(52)$ & $4(50)$ & $10(48)$ & 0.38 \\
\hline Anterior $\mathrm{PH}$ & $18(75)$ & $29(67)$ & $19(76)$ & $4(50)$ & $12(67)$ & 0.66 \\
\hline Complete PH & $16(64)$ & $21(48)$ & $12(48)$ & $3(38)$ & $8(40)$ & 0.49 \\
\hline Secondary neoplasms & $2(8)$ & $11(25)$ & $6(24)$ & $1(13)$ & $1(5)$ & 0.12 \\
\hline Cardio- and/or cerebrovascular events & $2(8)$ & $8(18)$ & $4(16)$ & $2(25)$ & $4(19)$ & 0.71 \\
\hline
\end{tabular}

Data regarding visual acuity disorders and visual field defects were missing in 15 and 28 patients respectively. Weight status could not be determined in 16 patients. Data regarding pituitary hormone deficiencies were missing in 5 patients (GH in 4 patients, FSH/LH in 1 patient, TSH in 1 patient, anterior PH in 5 patients, and complete $\mathrm{PH}$ in 1 patient). Also 8 patients were too young to reliably assess gonadal function.

IQR, interquartile range; ${ }^{90}$ Yttrium, ${ }^{90}$ Yttrium brachytherapy; BMI, body mass index; GTR, gross total resection; RTx, radiotherapy; STR, subtotal resection; w/o, without.

and deaths not directly related to the craniopharyngioma itself occurred with similar frequencies after initial craniopharyngioma treatment with gross total resection, subtotal resection with or without radiotherapy, cyst aspiration and ${ }^{90}$ Yttrium brachytherapy $(P=0.90)$, as well as in patients with childhood- and adult-onset disease $(P=0.28)$.

\section{Discussion}

In this large single-centre study of patients with craniopharyngioma treated from 1980 onwards, we cross-sectionally analysed retrospectively collected data on a full spectrum of long-term health conditions. After a median follow-up of 13 years since presentation, we observed a similar pattern of long-term health effects after initial craniopharyngioma treatment with gross total resection, subtotal resection with or without radiotherapy, cyst aspiration and ${ }^{90}$ Yttrium brachytherapy. In general, long-term health conditions were more frequent in patients with childhood- compared with adult-onset craniopharyngioma.

Pituitary hormone deficiencies, visual disturbances and obesity were the most frequently observed long-term health conditions in our study. This is in concordance with other studies that reported prevalence rates of 78-100\% for pituitary hormone deficiencies, $49-87 \%$ for visual disturbances and $27-57 \%$ for obesity $(10,20,22$, $23,26,28,30,32)$. Notably, we observed a relatively high frequency of obstructive sleep apnoea (i.e. 9\%) in patients with craniopharyngioma. This is considerably higher than the prevalence of obstructive sleep apnoea in the Dutch general population, which is estimated to be $1-4 \%$ (38). Since only patients with suspected sleep disordered 
A

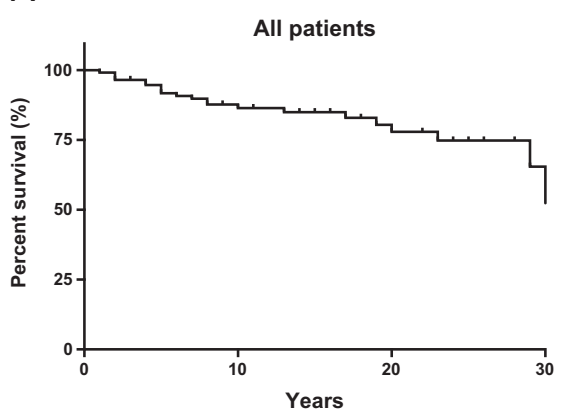

C Childhood-vs adult-onset

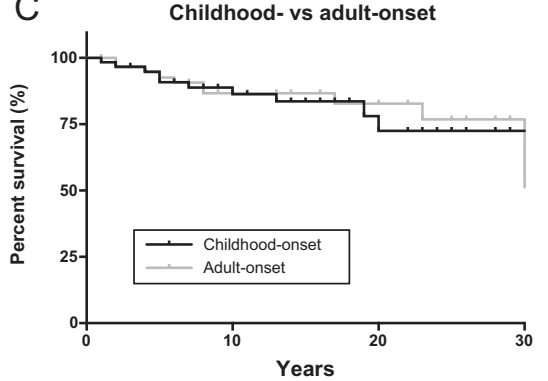

B

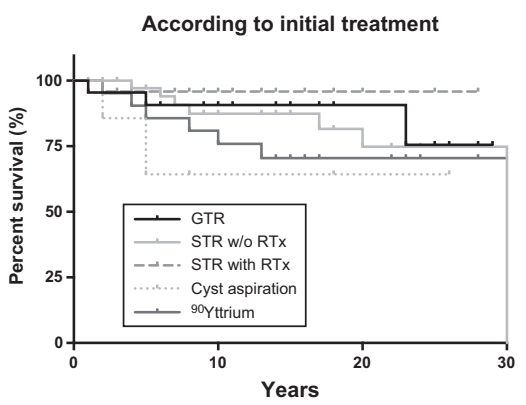

D

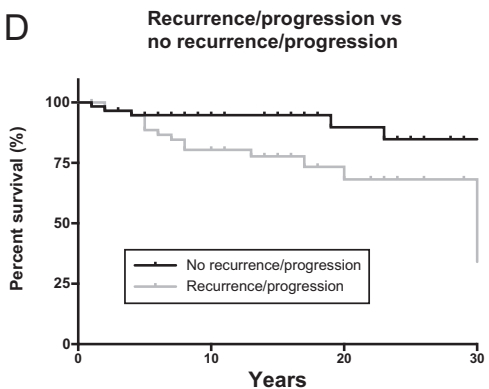

\section{Figure 2}

Overall survival in patients with craniopharyngioma. (A) All patients.

(B) According to initial craniopharyngioma treatment. (C) Patients with childhoodand adult-onset craniopharyngioma. (D) According to recurrence/progression status. ${ }^{90}$ Yttrium, ${ }^{90}$ Yttrium brachytherapy; GTR, gross total resection; RTX, radiotherapy; STR, subtotal resection; w/o, without. breathing were evaluated for obstructive sleep apnoea in our study, the observed frequency of $9 \%$ may even be an underestimation of the real prevalence of obstructive sleep apnoea in patients with craniopharyngioma. Only a few other studies compared long-term health conditions in patients with craniopharyngioma according to initial treatment approach $(17,35,36,37)$. In concordance with our results, these studies observed no important differences in long-term health effects after different initial craniopharyngioma treatment approaches. Lo et al. only observed a higher frequency of diabetes insipidus after initial craniopharyngioma treatment with neurosurgery compared with craniopharyngioma treatment without neurosurgery in 123 patients after a median follow-up of 9 years (35). Karavitaki et al. only observed more visual field defects after initial craniopharyngioma treatment with subtotal resection without radiotherapy compared with craniopharyngioma treatment with gross total resection or subtotal resection with radiotherapy in 121 patients after a mean follow-up of 9 years (36). Schoenfeld et al. only observed a higher rate of diabetes insipidus and panhypopituitarism after initial craniopharyngioma treatment with gross total resection compared with subtotal resection with or without radiotherapy in 122 patients after a median follow-up of 5 years (17). Merchant et al. only observed more diabetes insipidus and less hypogonadotropic hypogonadism after initial craniopharyngioma treatment with gross total resection compared with subtotal resection with radiotherapy in 30 patients after a median follow-up of 6 years (37).

In our study, long-term health conditions seemed to occur more frequently in patients with childhoodcompared with adult-onset craniopharyngioma. This may be explained by differences in tumour characteristics between patients with childhood- and adult-onset craniopharyngioma already present at diagnosis. In our study, patients with childhood-onset craniopharyngioma presented more often with multicystic tumours, as well as tumours associated with hydrocephalus and hypothalamic and/or third ventricle involvement compared with patients with adult-onset craniopharyngioma. In contrast, we observed significantly more cardio- and cerebrovascular events in patients with adult- compared with childhood-onset disease. This may be due to the significantly older age at follow-up of patients with adultonset craniopharyngioma. Aging has been established as an important risk factor for cardio- and cerebrovascular disease (39). Several other studies compared long-term health conditions between patients with childhoodand adult-onset craniopharyngioma $(28,30,36)$. In concordance with our results, Gautier et al. observed a significantly higher prevalence of diabetes insipidus and panhypopituitarism in patients with childhoodcompared with adult-onset disease in a cohort of 171 patients derived from two academic centres in France after a median follow-up of 12 years (30). In contrast, 


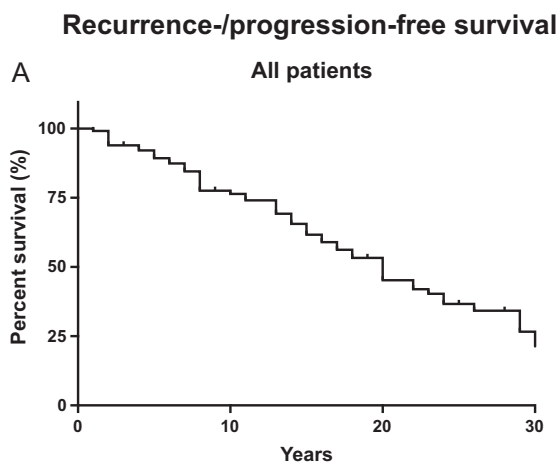

B According to initial treatment

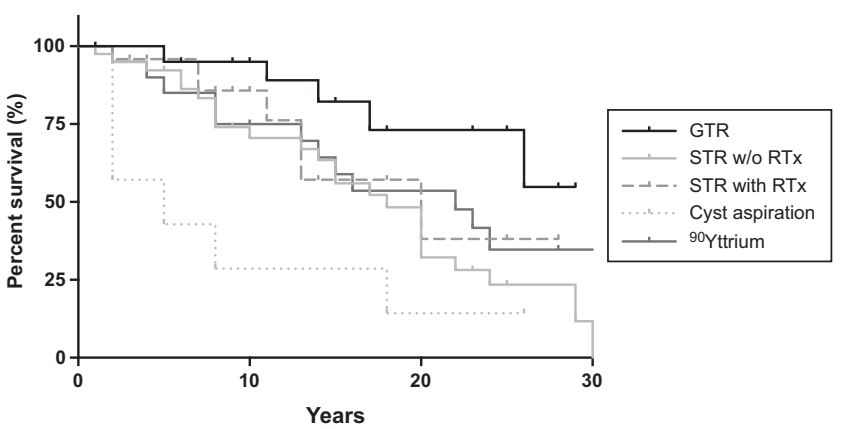

C Childhood- vs adult-onset

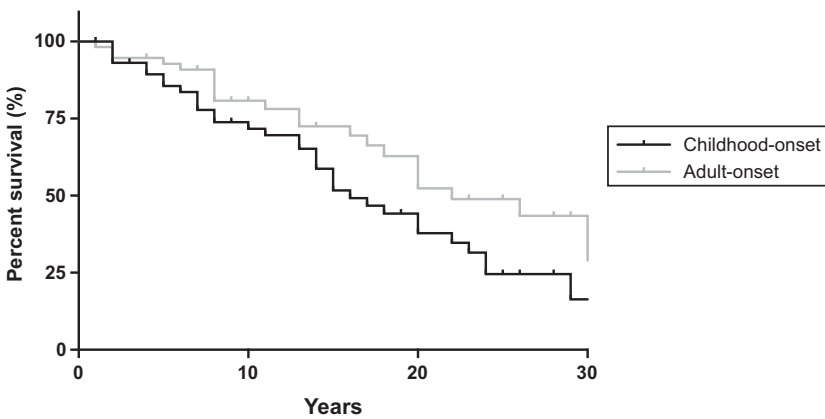

Figure 3

Recurrence-/progression-free survival in patients with craniopharyngioma. (A) All patients. (B) According to initial craniopharyngioma treatment. (C) Patients with childhoodand adult-onset craniopharyngioma. ${ }^{90}$ Yttrium, ${ }^{90}$ Yttrium brachytherapy; GTR, gross total resection; RTx, radiotherapy; STR, subtotal resection; w/o, without.

Kendall-Taylor et al. observed a significantly higher frequency of obesity in patients with adult- compared with childhood-onset craniopharyngioma. In their study, which included 393 growth hormone-deficient patients from the KIMS (Pfizer International Metabolic Database) who were followed for a mean 17 years, frequencies of any pituitary hormone deficiency and panhypopituitarism were similar between patients with childhood- and adult-onset disease (28). The aforementioned study by
Karavitaki et al. did not observe any significant differences in long-term health conditions between patients with childhood- and adult-onset craniopharyngioma, except for complete dependency for activities of daily living, which was more common in patients with adult-onset craniopharyngioma (36).

We observed 10-year overall and recurrence-/ progression-free survival rates after initial craniopharyngioma treatment of $85 \%$ and $74 \%$ respectively. This is comparable to other studies in patients with craniopharyngioma that reported 10 -year overall and recurrence-/progression-free survival rates between $40-95 \%$ and $44-76 \%$ respectively $(10,21,22$, $23,24,25)$. In our study, overall survival was similar after initial craniopharyngioma treatment with gross total resection, subtotal resection with or without radiotherapy, cyst aspiration and ${ }^{90}$ Yttrium brachytherapy. However, recurrence-/progression-free survival was significantly lower after initial craniopharyngioma treatment with cyst aspiration compared with the other therapeutic approaches. Two other studies also investigated overall and recurrence-/progression-free survival in patients with craniopharyngioma according to initial treatment approach $(34,36)$. The aforementioned study by Karavitaki et al. observed similar overall survival after initial craniopharyngioma treatment with gross total resection and subtotal resection with or without radiotherapy. However, recurrence-/progressionfree survival was significantly lower after initial craniopharyngioma treatment with subtotal resection without radiotherapy compared with gross total resection or subtotal resection with radiotherapy (36). A recent study by Rao et al. investigated 5-year overall survival in 697 patients with craniopharyngioma registered in the United States National Cancer Database. Overall survival was significantly lower after subtotal resection without radiotherapy compared with gross total resection or subtotal resection with radiotherapy (34). In our study, overall and recurrence-/progression-free survival were comparable in patients with childhood- and adult-onset craniopharyngioma. Other studies observed similar results $(24,30,36)$. However, the aforementioned study by Rao et al. observed significantly lower overall survival in patients with adult- compared with childhoodonset craniopharyngioma (34). Interestingly, patients with craniopharyngioma seem to have an increased mortality risk compared with patients with other pituitary diseases (21). This might be related to longterm health conditions caused by tumour and treatment (e.g. hypopituitarism and hypothalamic dysfunction), 
as well as to the locally aggressive behaviour of craniopharyngiomas and their tendency to recur after treatment $(21,22)$.

In our institution, initial craniopharyngioma treatment became less aggressive over time. This is in concordance with other studies $(4,20)$. We observed significantly lower risks of morbid obesity and severe neurological deficits after initial craniopharyngioma treatment in more recent decades. In contrast, we observed a significantly higher risk of recurrence/ progression in patients treated for craniopharyngioma in more recent decades.

Strengths of our study include the full spectrum of long-term health conditions investigated, as well as the relatively large number of patients treated with ${ }^{90}$ Yttrium brachytherapy assessed. Brachytherapy was first introduced as a treatment for craniopharyngioma in 1952 by Leksell and Liden, and involves the minimallyinvasive stereotactic application of beta-emitting isotopes in craniopharyngioma cysts (40). Subsequent radiation-induced destruction of epithelial cyst lining results in cyst shrinkage (41). Due to the short half-value depth of ${ }^{90}$ Yttrium in soft tissue, ${ }^{90}$ Yttrium brachytherapy is not effective for solid craniopharyngiomas (42). Only a few other studies investigated long-term health conditions after craniopharyngioma treatment with ${ }^{90}$ Yttrium brachytherapy $(42,43,44)$. These studies reported somewhat lower frequencies of long-term health effects compared with our study (55-90\% for pituitary hormone deficiencies, $61-90 \%$ for visual disturbances and $51 \%$ for obesity). This may be explained by a longer follow-up duration after ${ }^{90}$ Yttrium brachytherapy in our study (22 years vs 3-12 years in the other studies). Besides these strengths, some limitations should be considered when interpreting the results of our study. The retrospective nature of data collection may predispose to selection bias and missing data. However, due to the rarity of craniopharyngioma and the prolonged follow-up duration required to adequately study long-term health conditions, it seems unlikely that prospective observational studies with an adequate follow-up duration and a sufficient number of patients will become available in the near future. In addition, neuroimaging techniques were less sensitive earlier compared with more recent decades. This may have biased the assessment of the degree of craniopharyngioma resection, especially during earlier decades. Furthermore, our results on long-term neurocognitive and neuropsychological sequelae should be interpreted with caution, as cognitive impairments and behavioural changes were considered present if documented in the patient files and have not been formally tested. Moreover, we were unable to compare long-term health conditions according to histological subtype of craniopharyngioma (i.e. adamantinomatous or papillary), since these data were only available in a minority of patients.

In conclusion, our study demonstrates that despite encouraging survival rates, long-term health conditions are frequent in patients with craniopharyngioma. Initial gross total resection, subtotal resection with or without radiotherapy, cyst aspiration and ${ }^{90}$ Yttrium brachytherapy result in comparable long-term health effects. Patients with childhood-onset craniopharyngioma are generally more affected by long-term health conditions than patients with adult-onset disease. Craniopharyngioma should be regarded as a chronic condition requiring multidisciplinary management, both at diagnosis and during follow-up. Due to the high frequency of endocrine disorders, endocrinologists should play a pivotal role in the primary and follow-up care of patients with craniopharyngioma. Future studies on craniopharyngioma should focus on modifiable risk factors for long-term health conditions, as well as on therapeutic strategies that aim to reduce the long-term tumour- and treatment-related morbidity.

\section{Declaration of interest}

The authors declare that there is no conflict of interest that could be perceived as prejudicing the impartiality of this study.

\section{Funding}

This research did not receive any specific grant from any funding agency in the public, commercial or not-for-profit sector.

\section{Acknowledgements}

The authors would like to thank the physicians involved in the treatment and follow-up care of our patients.

\section{References}

1 Muller HL. Craniopharyngioma. Endocrine Reviews 201435 513-543. (doi:10.1210/er.2013-1115)

2 Nielsen EH, Feldt-Rasmussen U, Poulsgaard L, Kristensen LO, Astrup J, Jorgensen JO, Bjerre P, Andersen M, Andersen C, Jorgensen J et al. Incidence of craniopharyngioma in Denmark $(n=189)$ and estimated world incidence of craniopharyngioma in children and adults. Journal of Neuro-Oncology 2011104 755-763. (doi:10.1007/s11060011-0540-6)

3 Von Zenker AF. Enorme Zystenbildung im Gehirn, vom Hirnanhang ausgehend. Virchows Archiv 18572 454-466. (doi:10.1007/ bf01939537)

4 Barkhoudarian G \& Laws ER. Craniopharyngioma: history. Pituitary 201316 1-8. (doi:10.1007/s11102-012-0402-z) 
5 Karavitaki N. Management of craniopharyngiomas. Journal of Endocrinological Investigation 201437 219-228. (doi:10.1007/s40618013-0050-9)

6 Buchfelder M, Schlaffer SM, Lin F \& Kleindienst A. Surgery for craniopharyngioma. Pituitary 201316 18-25. (doi:10.1007/s11102012-0414-8)

7 Mortini P, Losa M, Pozzobon G, Barzaghi R, Riva M, Acerno S, Angius D, Weber G, Chiumello G \& Giovanelli M. Neurosurgical treatment of craniopharyngioma in adults and children: early and longterm results in a large case series. Journal of Neurosurgery $2011 \mathbf{1 1 4}$ 1350-1359. (doi:10.3171/2010.11.JNS10670)

8 Elliott RE, Hsieh K, Hochm T, Belitskaya-Levy I, Wisoff J \& Wisoff $\mathrm{JH}$. Efficacy and safety of radical resection of primary and recurrent craniopharyngiomas in 86 children. Journal of Neurosurgery Pediatrics 20105 30-48. (doi:10.3171/2009.7.PEDS09215)

9 Zhang YQ, Ma ZY, Wu ZB, Luo SQ \& Wang ZC. Radical resection of 202 pediatric craniopharyngiomas with special reference to the surgical approaches and hypothalamic protection. Pediatric Neurosurgery 200844 435-443. (doi:10.1159/000172965)

10 Du C, Feng CY, Yuan XR, Liu Q, Peng ZF, Jiang XJ, Li XJ, Xiao GL, Li YF \& Xiong T. Microsurgical management of craniopharyngiomas via an unilateral subfrontal approach - a retrospective study of 177 continuous cases. World Neurosurgery $201690454-468$. (doi:10.1016/j.wneu.2016.03.002)

11 Bal E, Oge K \& Berker M. Endoscopic endonasal transsphenoidal surgery, a reliable method for treating primary and recurrent/residual craniopharyngiomas: nine years of experience. World Neurosurgery 201694 375-385. (doi:10.1016/j.wneu.2016.07.004)

12 Xing H, Xing H, Hui P \& Yang B. Removal of craniopharyngioma via fronto-basal interhemispheric approach. Oncology Letters 201612 147-149. (doi:10.3892/ol.2016.4555)

13 Cheng J, Shao Q, Pan Z \& You J. Analysis and long-term follow-up of the surgical treatment of children with craniopharyngioma. Journal of Craniofacial Surgery 201627 e763-e766. (doi:10.1097/ SCS.0000000000003176)

14 Moussazadeh N, Prabhu V, Bander ED, Cusic RC, Tsiouris AJ, Anand VK \& Schwartz TH. Endoscopic endonasal versus open transcranial resection of craniopharyngiomas: a case-matched single-institution analysis. Neurosurgical Focus 201641 E7. (doi:10.3171/2016.9.FO CUS16299)

15 Morisako H, Goto T, Goto H, Bohoun CA, Tamrakar S \& Ohata K. Aggressive surgery based on an anatomical subclassification of craniopharyngiomas. Neurosurgical Focus 201641 E10. (doi:10.3171/2 016.9.FOCUS16211)

16 Winkfield KM, Tsai HK, Yao X, Larson E, Neuberg D, Pomeroy SL, Ullrich NJ, Cohen LE, Kieran MW, Scott RM et al. Long-term clinical outcomes following treatment of childhood craniopharyngioma. Pediatric Blood and Cancer 201156 1120-1126. (doi:10.1002/ pbc.22884)

17 Schoenfeld A, Pekmezci M, Barnes MJ, Tihan T, Gupta N, Lamborn KR, Banerjee A, Mueller S, Chang S, Berger MS et al. The superiority of conservative resection and adjuvant radiation for craniopharyngiomas. Journal of Neuro-Oncology 2012108 133-139. (doi:10.1007/s11060-012-0806-7)

18 Schubert T, Trippel M, Tacke U, van Velthoven V, Gumpp V, Bartelt S, Ostertag C \& Nikkhah G. Neurosurgical treatment strategies in childhood craniopharyngiomas: is less more? Child's Nervous System 200925 1419-1427. (doi:10.1007/s00381-009-0978-4)

19 Puget S, Garnett M, Wray A, Grill J, Habrand JL, Bodaert N, Zerah M, Bezerra M, Renier D, Pierre-Kahn A et al. Pediatric craniopharyngiomas: classification and treatment according to the degree of hypothalamic involvement. Journal of Neurosurgery 2007 106 3-12. (doi:10.3171/ped.2007.106.1.3)

20 Tan TS, Patel L, Gopal-Kothandapani JS, Ehtisham S, Ikazoboh EC, Hayward R, Aquilina K, Skae M, Thorp N, Pizer B et al. The neuroendocrine sequelae of paediatric craniopharyngioma: a 40 year meta-data analysis of 185 cases from three UK centres. European Journal of Endocrinology 2017176 359-369. (doi:10.1530/EJE-16-0812)

21 Sherlock M, Ayuk J, Tomlinson JW, Toogood AA, Aragon-Alonso A, Sheppard MC, Bates AS \& Stewart PM. Mortality in patients with pituitary disease. Endocrine Reviews 201031 301-342. (doi:10.1210/ er.2009-0033)

22 Olsson DS, Andersson E, Bryngelsson IL, Nilsson AG \& Johannsson G. Excess mortality and morbidity in patients with craniopharyngioma, especially in patients with childhood onset: a population-based study in Sweden. Journal of Clinical Endocrinology and Metabolism 2015100 467-474. (doi:10.1210/jc.2014-3525)

23 Lee CC, Yang HC, Chen CJ, Hung YC, Wu HM, Shiau CY, Guo WY, Pan DH, Chung WY \& Liu KD. Gamma Knife surgery for craniopharyngioma: report on a 20-year experience. Journal of Neurosurgery 2014121 (Supplement) 167-178. (doi:10.3171/2014.8.GKS141411)

24 Pan J, Qi S, Liu Y, Lu Y, Peng J, Zhang X, Xu Y, Huang GL \& Fan J. Growth patterns of craniopharyngiomas: clinical analysis of 226 patients. Journal of Neurosurgery Pediatrics 201617 418-433. (doi:10.3171/2015.7.PEDS14449)

25 Hoffmann A, Warmuth-Metz M, Lohle K, Reichel J, Daubenbuchel AM, Sterkenburg AS \& Muller HL. Fusiform dilatation of the internal carotid artery in childhood-onset craniopharyngioma: multicenter study on incidence and long-term outcome. Pituitary 201619 422-428. (doi:10.1007/s11102-016-0722-5)

26 Elliott RE, Jane JA Jr \& Wisoff JH. Surgical management of craniopharyngiomas in children: meta-analysis and comparison of transcranial and transsphenoidal approaches. Neurosurgery 201169 630-643; discussion 643. (doi:10.1227/NEU.0b013e31821a872d)

27 Honegger J, Buchfelder M \& Fahlbusch R. Surgical treatment of craniopharyngiomas: endocrinological results. Journal of Neurosurgery 199990 251-257. (doi:10.3171/jns.1999.90.2.0251)

28 Kendall-Taylor P, Jonsson PJ, Abs R, Erfurth EM, Koltowska-Haggstrom M, Price DA \& Verhelst J. The clinical, metabolic and endocrine features and the quality of life in adults with childhood-onset craniopharyngioma compared with adult-onset craniopharyngioma. European Journal of Endocrinology 2005152 557-567. (doi:10.1530/ eje.1.01877)

29 Sterkenburg AS, Hoffmann A, Gebhardt U, Warmuth-Metz M, Daubenbuchel AM \& Muller HL. Survival, hypothalamic obesity, and neuropsychological/psychosocial status after childhood-onset craniopharyngioma: newly reported long-term outcomes. NeuroOncology 201517 1029-1038. (doi:10.1093/neuonc/nov044)

30 Gautier A, Godbout A, Grosheny C, Tejedor I, Coudert M, Courtillot C, Jublanc C, De Kerdanet M, Poirier JY, Riffaud L et al. Markers of recurrence and long-term morbidity in craniopharyngioma: a systematic analysis of 171 patients. Journal of Clinical Endocrinology and Metabolism 201297 1258-1267. (doi:10.1210/jc.2011-2817)

31 Wijnen M, Olsson DS, van den Heuvel-Eibrink MM, Wallenius V, Janssen J, Delhanty PJ, van der Lely AJ, Johannsson G \& Neggers S. Efficacy and safety of bariatric surgery for craniopharyngiomarelated hypothalamic obesity-A matched case-control study with two years of follow-up. International Journal of Obesity 201641 210-216. (doi:10.1038/ijo.2016.195)

32 Muller HL, Bueb K, Bartels U, Roth C, Harz K, Graf N, Korinthenberg R, Bettendorf M, Kuhl J, Gutjahr P et al. Obesity after childhood craniopharyngioma - German multicenter study on pre-operative risk factors and quality of life. Klinische Pädiatrie 2001213 244-249. (doi:10.1055/s-2001-16855)

33 Smith TR, Cote DJ, Jane JA Jr \& Laws ER Jr. Physiological growth hormone replacement and rate of recurrence of craniopharyngioma: the Genentech National Cooperative Growth Study. Journal of Neurosurgery Pediatrics 201618 408-412. (doi:10.3171/2016.4.PEDS16112)

34 Rao YJ, Hassanzadeh C, Fischer-Valuck B, Chicoine MR, Kim AH, Perkins SM \& Huang J. Patterns of care and treatment outcomes of patients with Craniopharyngioma in the national cancer database. 
Journal of Neuro-Oncology 2016132 109-117. (doi:10.1007/s11060016-2342-3)

35 Lo AC, Howard AF, Nichol A, Sidhu K, Abdulsatar F, Hasan H \& Goddard K. Long-term outcomes and complications in patients with craniopharyngioma: the British Columbia cancer agency experience. International Journal of Radiation Oncology, Biology, Physics 201488 1011-1018. (doi:10.1016/j.ijrobp.2014.01.019)

36 Karavitaki N, Brufani C, Warner JT, Adams CB, Richards P, Ansorge $\mathrm{O}$, Shine B, Turner HE \& Wass JA. Craniopharyngiomas in children and adults: systematic analysis of 121 cases with long-term follow-up. Clinical Endocrinology 200562 397-409. (doi:10.1111/j.13652265.2005.02231.x)

37 Merchant TE, Kiehna EN, Sanford RA, Mulhern RK, Thompson SJ, Wilson MW, Lustig RH \& Kun LE. Craniopharyngioma: the St. Jude Children's Research Hospital experience 1984-2001. International Journal of Radiation Oncology, Biology, Physics 200253 533-542. (doi:10.1016/S0360-3016(02)02799-2)

38 Damen-van Beek Z, Lucassen PL, Gorgels W, Smelt AF, Knuistingh Neven A \& Bouma M. The NHG guideline 'sleep problems and sleeping pills'. Nederlands Tijdschrift voor Geneeskunde 2015159 A8679.
39 Sniderman AD \& Furberg CD. Age as a modifiable risk factor for cardiovascular disease. Lancet 2008371 1547-1549. (doi:10.1016/ S0140-6736(08)60313-X)

40 Leksell L \& Liden K. A therapeutic trial with radioactive isotopes in cystic brain tumour. In Radioisotope Techniques, Volume 1: Medical and Physiological Applications. pp. 1-4 Oxford: HM Stationary Office 1952.

41 Szeifert GT, Balint K, Sipos L, Sarker MH, Czirjak S \& Julow J. Pathological findings in cystic craniopharyngiomas after stereotactic intracavitary irradiation with yttrium-90 isotope. Progress in Neurological Surgery 200720 297-302. (doi:10.1159/000100173)

42 Van den Berge JH, Blaauw G, Breeman WA, Rahmy A \& Wijngaarde R. Intracavitary brachytherapy of cystic craniopharyngiomas. Journal of Neurosurgery 199277 545-550. (doi:10.3171/jns.1992.77.4.0545)

43 Voges J, Sturm V, Lehrke R, Treuer H, Gauss C \& Berthold F. Cystic craniopharyngioma: long-term results after intracavitary irradiation with stereotactically applied colloidal beta-emitting radioactive sources. Neurosurgery 199740 263-269; discussion 269-270. (doi:10.1097/00006123-199702000-00006)

44 Julow JV. Intracystic irradiation for craniopharyngiomas. Pituitary 201316 34-45. (doi:10.1007/s11102-012-0442-4)

Received 19 January 2017

Revised version received 16 March 2017

Accepted 21 March 2017 